\title{
Traumatic posterior tibial pseudoaneursym: a rare complication repaired conventionally
}

Farzad Amiri MD ${ }^{1}$, Zach Sandford MD ${ }^{1}$, Constantinous Constantinou MD ${ }^{1}$

Author Affiliations:

1. Joan C Edwards School of Medicine, Marshall University, Huntington, West Virginia

The authors have no financial disclosures to declare and no conflicts of interest to report.

\section{Corresponding Author:}

Farzad Amiri MD

Marshall University Joan C. Edwards School of Medicine

Huntington, West Virginia

Email: lemacfar@gmail.com 


\section{Abstract}

Posterior tibial artery pseudoaneurysms are extremely rare. They commonly present as a late complication of trauma or iatrogenic injury. Diagnosis can be elusive. Treatment has been described in the context of open primary repair, endovascular stenting, and coil embolization. There has been increasing interest in the use of endovascular treatment options for this type of injury. If left untreated, pseudoaneurysms can rupture, partially thrombose, expand and cause adjacent local compression and compartment syndrome. The following case presented is of a young gentleman with a posterior tibial pseudoaneurysm incidentally discovered after stepping on glass and the management of his posterior tibial pseudoaneurysm.

\section{Keywords}

Posterior Tibial Pseudoaneursym, Vascular Surgery, Aneurysm, Trauma, pseudoaneurysm

\section{Introduction}

Posterior tibial artery pseudoaneurysms are extremely rare with less than thirty reported cases published in the literature. Like pseudoaneurysms in other locations, different treatment modalities have been described in the literature. Treatment has been described in the context of open primary repair, endovascular stenting, and coil embolization. There has been increasing interest in the use of endovascular treatment options for this type of injury. If left untreated, pseudoaneurysms can rupture, partially thrombose, expand and cause adjacent local compression and compartment syndrome. ${ }^{1,2}$ The following case presented is of a young gentleman with a posterior tibial pseudoaneurysm incidentally discovered after stepping on glass and the management of his posterior tibial pseudoaneurysm.

\section{Presentation}

A 20-year-old male presents to our emergency department with pain to his right ankle with pulsatile mass. Patient reportedly stepped on broken glass with a small laceration that was closed primarily by the emergency physician five days prior. He was seen in the emergency department on our presentation for a routine wound check. Physical exam revealed a four centimeter pulsatile mass posterior to the medial malleolus with pain and ecchymosis to that area. There was no active bleeding. The wound was closed five days prior and at that time there were no reports of a pulsatile mass. Duplex ultrasonography confirmed physical exam findings for presence of a large $4 \mathrm{~cm}$ posterior tibial artery pseudoaneurysm (figures 1,2,3) The large and expanding nature of this pseudoaneurysm prompted surgical intervention. He underwent an aneurysm excision and primary arterial reconstruction. During operation, the pseudoaneurysm had a short neck with irregular edges involving greater than $50 \%$ of the circumference of the artery wall. The diseased artery was resected, approximately $2 \mathrm{~cm}$, mobilized and re-anastomosed with beveled ends. The use of 7-0 prolene was used during this anastomosis. He had an uneventful postoperative course and was discharged on a short course of aspirin. He was seen in clinic post operatively and clinically has no sequela from the injury. 


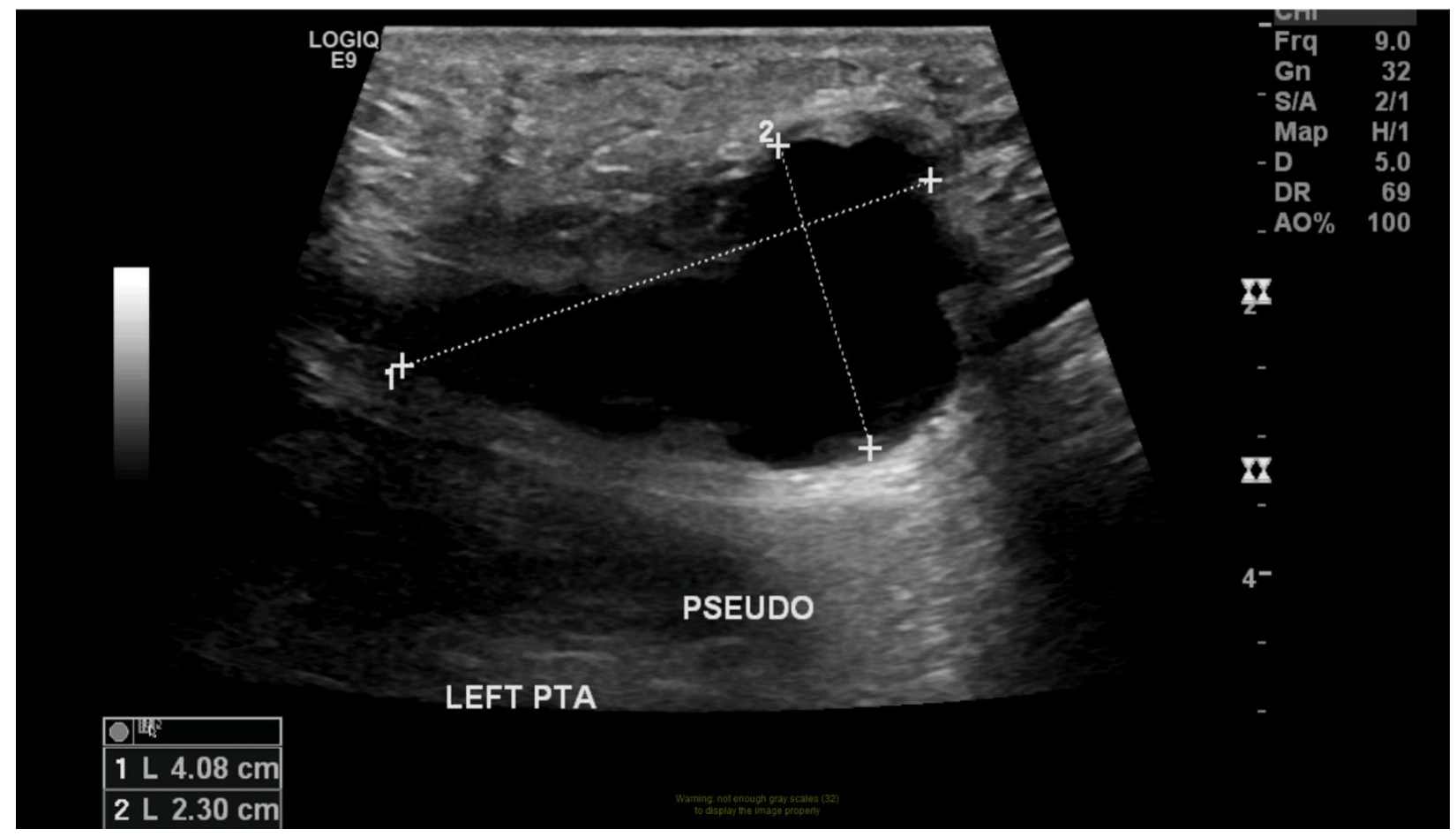

Figure 1

Figure 1 demonstrates the patient's pseudo aneurysm along with its dimensions. This was using a standard high quality ultrasound device. 


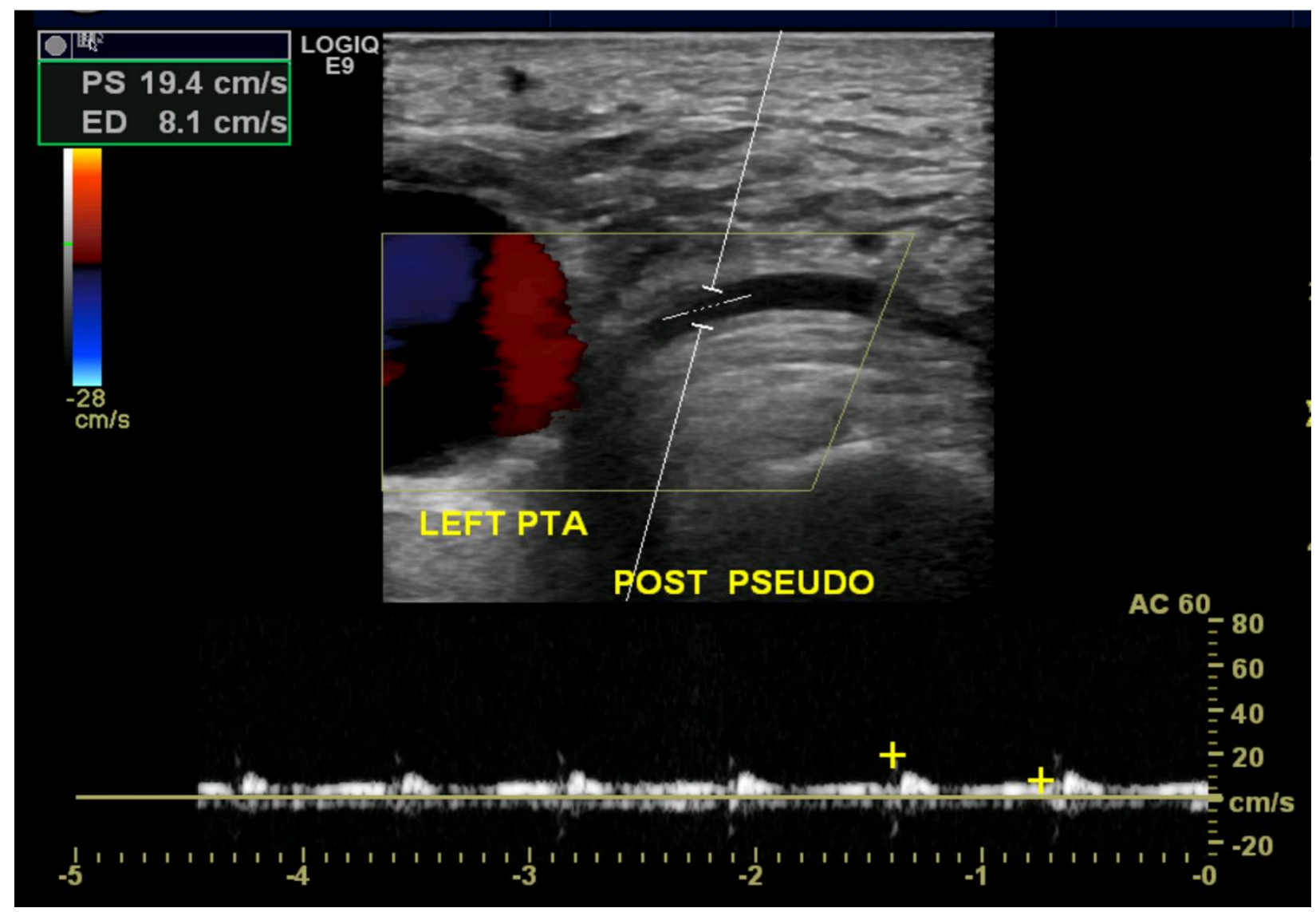

Figure 2

Figure 2 demonstrates the artery distal to the pseudo aneurysm. At the bottom of the image, you can see Doppler wave forms showing that blood flow exists distal to this. Additionally to the right of the image, the pseudo aneurysm exists and using color sonography, it demonstrates blood flow within the pseudo aneurysm sac. 


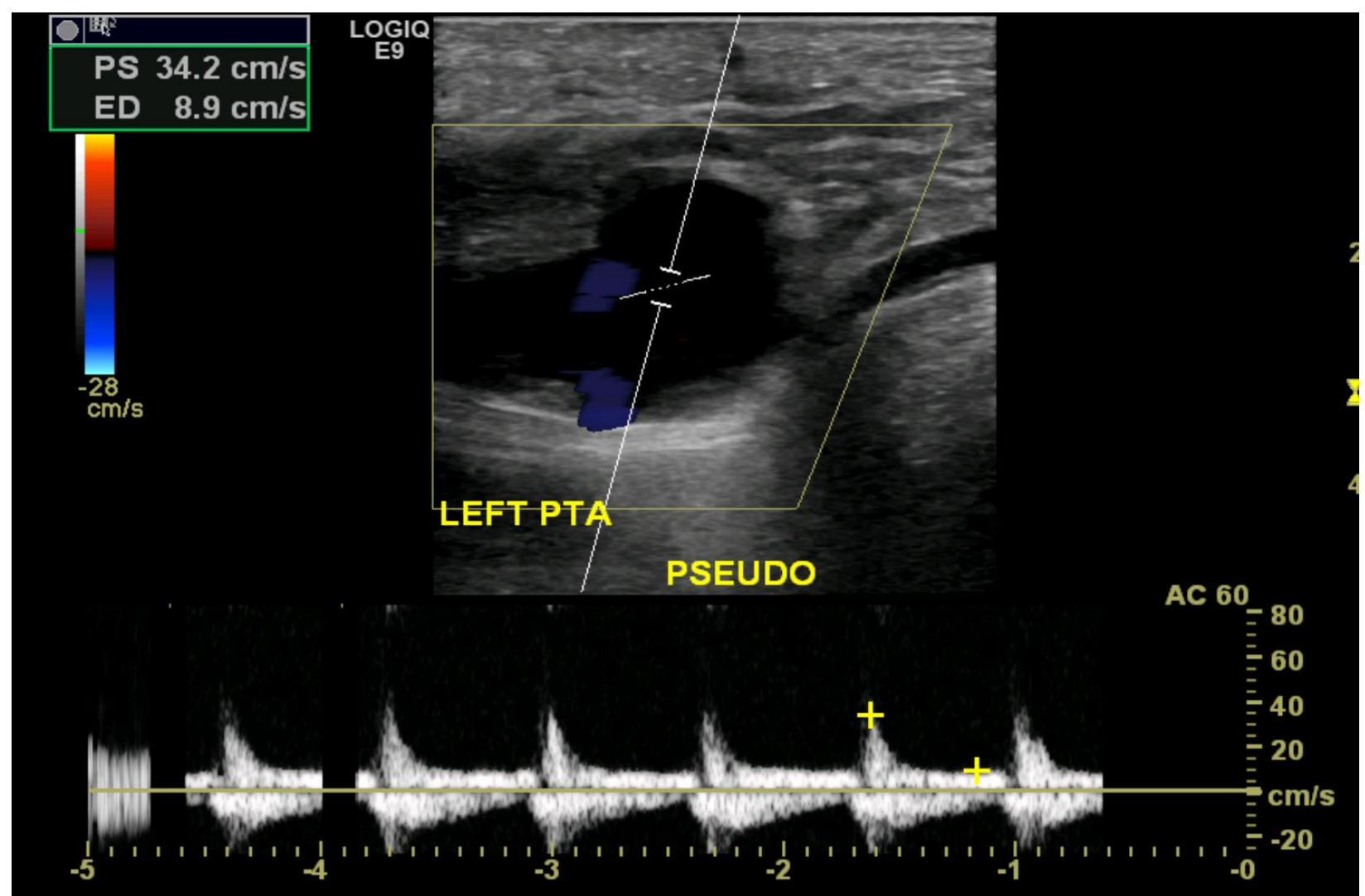

Figure 3

Figure 3 demonstrates Doppler waveforms (bottom of image) within the pseudo aneurysm sac. 


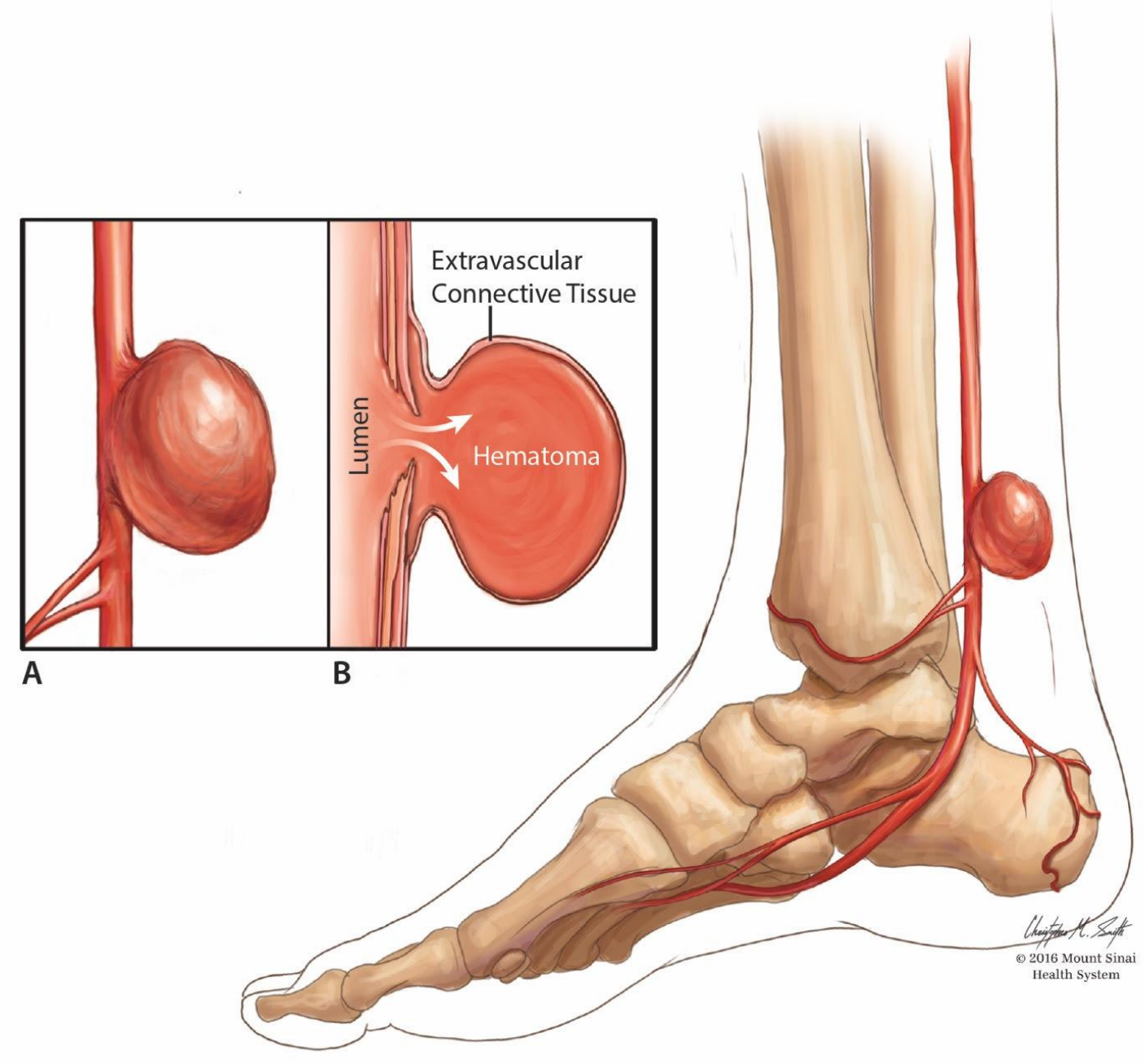

Figure 4

Figure 4 is an artist's depiction of a posterior tibial pseudo aneurysm. 


\section{Discussion}

Posterior tibial pseudoaneurysms are rare, with only twenty-six reported cases in the literature. Commonly they present as a late complication of trauma or iatrogenic injury. They usually present as a pulsatile mass with or without neurologic complaint. Diagnosis can be elusive due to the rarity of the condition, presence of minimal or atypical complaints, usually with a normal extremity pulse exam.

The best initial imaging modality is duplex ultrasonography which can demonstrate to and fro flow within the false aneurysm, size of aneurysm sac, and distal blood flow. A detailed history and physical examination along with ultrasound sonography is sufficient for diagnosis of a posterior tibial pseudoaneurysm.

The treatment options for a posterior tibial pseudoaneurysm vary. Traditionally, an open aneurysm excision with primary anastomosis or ligation would be adequate treatment. The decision on whether to ligate or anastomose the posterior tibial artery is dependent on the patient's comorbidities, vascular status, and whether collateral flow is present. Excision remains the most common treatment modality, however novel approaches such as thrombin injection, endovascular stenting and coil embolization have been described in the literature. ${ }^{3}$ The utility of these in the younger adult population is still experimental; with insufficient data on long term outcomes, concerns remain with endovascular stenting below the popliteal and patency. This patient presented with a rapidly expanding pseudoaneurysm as the symptoms were not present on initial ER evaluation. The use of open versus endovascular approaches have been described and can be applied in specific situations and availability of resources. ${ }^{4}$

The use of aspirin therapy is also debatable. While there is no published data on patency rates for the use of antiplatelet therapy on posterior tibial artery primary anastomosis, many have looked retrospectively at the data. A recent Cochrane review of 5683 patients in multiple studies concluded a benefit of antiplatelet use in patients with bypass grafts. ${ }^{5}$ They did not however see a benefit in autologous vein bypasses. In this patient, we felt that the risk and benefit profile favored usage of aspirin therapy for a total of 90 days post operatively. There were no complications reported in the global post-operative period. 


\section{References}

1. Skudder PA, Gelfand ML, Blumenberg RM, Fulco J. Tibial artery false aneurysm: uncommon result of blunt injury occurring during athletics. Ann Vasc Surg. 1999;13:589-91.

2. Breen T, Brumback R. Posterior tibial artery pseudoaneurysm following tibial fracture: a case report. Orthopedics. 1985;8:993-7.

3. Joglar F, Kabutey NK, Maree A, Farber A. The role of stent grafts in the management of traumatic tibial artery pseudoaneurysms: case report and review of the literature. Vasc Endovascular Surg. 2010;44:407-9.

4. Ricotta JJ. False aneurysm and arteriovenous fistula. In: Cameron JL, editor. Current Surgical Therapy. 4th ed. St. Louis MO: Mosby; 1992. pp. 681-61.

5. Benedi R. Antiplatelet agents for preventing thrombosis after peripheral arterial bypass surgery. Cochrane Database Syst. Rev. 2015. 